\title{
Antropología, "raza" y población en Cuba en el último cuarto del siglo XIX*
}

\author{
Armando García González \\ Centro de Estudios de Historia de la Ciencia \\ y la Tecnología. La Habana \\ Consuelo Naranjo Orovio \\ Centro de Estudios Históricos, CSIC. Madrid
}

El análisis del discurso racial emanado desde las ciencias antropológicas, médicas y biológicas revela la formación de categorías raciales que hacen que la "raza" no sólo sea una categoría social y cultural, así como que el racismo no sea un fenómeno coyuntural. El estudio de estos discursos científicos o pseudocientíficos y los instrumentos conceptuales que los sustentaron, nos ayudará a comprender por qué la "raza" ha sido siempre un factor recurrente en Cuba, al que se le ha instrumentalizado desde la política, la ciencia y la cultura, unas veces en aras de la integridad nacional y de la defensa de la soberanía y otras como medio de control de los sectores menos favorecidos. En los últimos años del siglo XIX la "raza" fue considerada por unos como baluarte del poder español en la isla, mientras que otros la valoraron como el bastión de la formación de la nueva sociedad.

$\mathrm{Al}$ abordar el tema de la discriminación racial, del racismo tanto cultural como social y/o científico, diferenciado por algunos autores, nuestro propósito es constatar que éste no es un fenómeno coyuntural, que obedezca a determinadas situaciones económicas, sociales, políticas o culturales. $\mathrm{Si}$ bien en determinados momentos se producen acontecimientos que refuerzan manifestaciones o actitudes racistas y, en definitiva, la discriminación, el racismo -entendiendo por éste la marginación de un sector social en función del color de la piel- no es coyuntural al estar afianzado en las culturas y en las sociedades a través de creencias, mitos, actitudes y teorías pseudocientíficas que con mayor o menor intensidad aparecen en determinados momentos. Este hecho, que hace que el racismo subyazca en las sociedades, se ve reforzado por las relaciones de poder y de clase.

Considerando a la "raza" como una construcción no sólo social, sino cultural, política y científica, nuestro propósito es estudiar las fuentes y teorías científicas que subyacen en los discursos de políticos e intelectuales, y que, a menudo, es a partir de éstas que se establecen con mayor "autoridad científica" las diferencias entre las distintas poblaciones, hasta llegar a con-

* Trabajo realizado dentro de los Proyectos PB94-0060 y PB96-0868 (DIGICYT) 
formar un cuerpo conceptual que permite establecer una rígida jerarquización biológica que subyace en las culturas y sociedades. Dicha jerarquización, según los momentos históricos, se manifiesta de formas diferentes, como categorías culturales, sociales, etc. Partiendo de esto, la "raza" es, además, una categoría social y una construcción política y cultural. ${ }^{1}$

La "raza" en Cuba siempre ha sido un tema recurrente al que acudieron distintos sectores de la elite y la población en aquellos momentos en los que el orden establecido parecía tambalearse. El miedo a la africanización de la isla, los deseos de "blanquear" el país y las tentativas de consolidar una nación con coherencia étnica y cultural, equivalente a un país "blanco", encontraron las diferentes soluciones, que según los momentos adoptaron, en las teorías científicas y creencias populares que establecían fuertes desigualdades culturales y sociales entre los individuos según su tonalidad. La antropología, fundamentalmente física, nos ayudará a adentrarnos en los prejuicios raciales esgrimidos en el siglo XIX, sobre todo a partir del último cuarto del siglo, y que continuaron presentes en las décadas siguientes. ${ }^{2}$

1 Young, Robert, J.C.: Colonial Desire. Hybridity in the Theory, Culture and Race, London and New York, 1995. La utilización de la ciencia, sobre todo de los conceptos procedentes de la medicina, antropología y biología, para demostrar la superioridad de unos pueblos ("razas") sobre otros la hemos abordado en otros estudios sobre el siglo XIX y XX: Naranjo Orovio, Consuelo, y García González, Armando: Inmigración y racismo en Cuba en el siglo XIX, Madrid, 1996; Medicina y racismo en Cuba. La ciencia ante la inmigración canaria, siglo XX, La Laguna, Tenerife, 1996. En otra línea y objetivos de análisis se encuentran los últimos estudios historiográficos que inciden en el papel desempeñado por los afrocubanos en las luchas de independencia cubana. Dentro de estos estudios interesantes, y centrados en el caso de Cuba, hay que destacar los siguientes: Helg, Aline: Our Rightful Share. The Afro-Cuban Struggle for Equality, 1886-1912, North Carolina, 1995. Zeuske, Michael: "El poder discreto de los exesclavos: "raza", clientelas y poder nacional en Cuba 1895-1908", ponencia presentada en el XI Congreso Internacional de AHILA, Liverpool, 17-22 de septiembre de 1996; véase el Dossier publicado en Historia Social, Valencia, núm. 22, 1995, sobre "Raza y racismo": Scott, Rebecca: "Relaciones de clase e ideologías raciales: acción rural colectiva en Louisiana y Cuba, 1865-1912”, Historia Social, núm. 22, 1995, págs. 127-149. Ferrer, Ada: "Esclavitud, ciudadanía y los límites de la nacionalidad cubana: la guerra de los diez años, 1868-1878”, Historia Social, núm. 22, 1995, págs. 101-126. Casanovas, Joan: "The Cuban Labor Movement of the 1860s and Spain's Search for a New Colonial Policy", Cuban Studies, núm. 25, 1995, págs. 83-99; Casaus, Marta: Guatemala: linaje y racismo, Costa Rica, 1995.

2 Algunas investigaciones recientes se centran en la politización de la "línea de color" y en la utilización de los prejuicios raciales en la definición de la ciudadanía: Irurozqui, Marta: "La amenaza chola. La participación popular en las elecciones bolivianas, 1900-1930”, Revista Andina, núm. 26, Cusco, 1995, págs. 357-388; "Ebrios, vagos y analfabetos. El sufragio restringido en Bolivia, 18261952”, Revista de Indias, núm. 208, Madrid, 1996, págs. 697-742. Ferrer, Ada: To Make a Free Nation. Race and Struggle for Independence in Cuba, Michigan, 1995. La Fuente, Alejandro de: "Race and Inequality in Cuba, 1899-1981", Journal of Contemporary History, 30:1, 1995, págs. 131-168; "Negros y electores: desigualdad y políticas raciales en Cuba, 1900-1930". Naranjo, Consuelo, Puig-Samper, Miguel Angel y García Mora, Luis M. (eds.): La Nación Soñada: Cuba, Puerto Rico y Filipinas ante el 98, Madrid, 1996, págs. 163-177; Scott, Rebecca: “ 'The lower class of whites' and 'the negro element': race, social identity, and politics in Central Cuba, 1899-1909”, Naranjo, Puig-Samper y García Mora (eds.): La Nación Soñada..., págs. 179-191. 


\section{La antropología en Cuba y el discurso racial científico}

Nuestro interés se centra en el análisis del discurso racial emanado desde la ciencia y los instrumentos conceptuales que sustentaron dicho discurso, ya que consideramos que, independientemente del momento, de las manifestaciones del racismo y de la utilización de la "raza", bajo todo ello permanecen las teorías científicas (categorías biológicas), que son las que nutren y dan contenido a las categorías sociales y culturales raciales. ${ }^{3}$

\section{La Real Academia de Ciencias Médicas, Físicas y Naturales de la Habana}

La creación de esta Academia y de la Sociedad Antropológica de la Isla de Cuba supuso la institucionalización de algunas ciencias, como la medicina, antropología, zoología y biología, que en esos momentos contaban con gran prestigio en Europa y Estados Unidos. ${ }^{4}$

En 1861 tuvo lugar en La Habana la fundación de la Real Academia de Ciencias Médicas, Físicas y Naturales, que contó con los Anales, a partir de 1864, como órgano de difusión de los debates que en su seno tenían lugar. Estos debates estuvieron focalizados en aspectos relacionados con la anatomía y patología de las "razas"; por un lado se ocupó de investiga-

3 La importancia de la "raza" en la formación de las naciones en América Latina ha sido tema de investigación reciente de algunos historiadores, véanse los trabajos pioneros de Graham, Richard: The Idea of Race in Latin America, Texas, 1990; Quijada Mauriño, Mónica: "De Perón a Alberdi: selectividad étnica y construcción nacional en la política inmigratoria argentina", Revista de Indias, Madrid, 1992, Vol. LII, núms. 194-195, págs. 867-888; "En torno al pensamiento racial en Hispanoamérica", EIAL, 3:1, 1992, págs. 109-130; "Los orígenes indoeuropeos de los Incas, o los usos de la Historia en el siglo XIX", González González, Enrique (coord.): Historia y Universidad. Homenaje a Lorenzo Luna, México, 1996, págs. 163-185; Wade, Peter: Blackness and Race Mixture: The Dynamics of Racial Identity in Colombia, Baltimore, 1993; Wright, W.: Café con leche: Race, Class and National Image in Venezuela, Texas, 1990; Needell, Jeffrey D.: "History, Race, and the State in the Thought of Oliveira Viana", HAHR 75:1, 1995, págs. 1-30; Naranjo Orovio, Consuelo: "En búsqueda de lo nacional: migraciones y racismo en Cuba (1880-1910)", Naranjo, Puig-Samper y García Mora (eds.): $L a$ Nación Soñada..., págs. 149-162; García Mora, Luis M. y Naranjo, Consuelo: "Intelectualidad criolla y nación en Cuba, 1878-1898”, Studia Historica, Salamanca, 1997 (en prensa).

4 El positivismo francés y la antropología norteamericana fueron las corrientes que mayor influencia ejercieron en los medios científicos cubanos. El desarrollo de la antropología en Francia ha sido estudiado en distintos trabajos por Elvira Arquiola; véase Arquiola, E.: "Racismo y antropología en Francia (1859-1880)", en Albarracín, Agustín, López Piñero, José M.a , y Graniel, L. S.: Medicina e historia, Madrid, 1980, págs. 235-249. Para el caso del desarrollo de la antropología en Estados Unidos, Peset Reig, José Luis: Ciencia y marginación. Sobre negros, locos y criminales, Barcelona, 1983. 
ciones sobre inmunidad y aclimatación de los distintos pueblos, y, por otro, se centró en estudios puramente antropológicos, a partir de los cuales se pudiera determinar la "raza" a la cual pertenecía un individuo, en los casos en los que, como era habitual, se solicitaba a la Academia que así lo dictaminase.

La relación estrecha entre la medicina y la geografía, debido al peso de la tradición médica en los conocimientos y aplicación de la geografía, determinó la concepción que de determinadas "razas", países y climas se tenía, sobre su salubridad o no, sobre la inmunidad a ciertas enfermedades y sobre las posibilidades de aclimatación a distintas latitudes. En el trabajo elaborado por M. Dupierris, Memoria sobre la topografía médica de La Habana y sus alrededores y sobre el estudio físico y natural de los colonos asiáticos, de 1857, se maneja la teoría hipocrática, que establecía una estrecha relación entre la geografía y la salud de los individuos, y en concreto entre el clima, la atmósfera, los vientos, el agua y la existencia de epidemias. ${ }^{5}$

La carestía del esclavo africano y la introducción de chinos, a partir de 1847, volvió a reavivar la polémica sobre la idoneidad de los distintos pueblos para aclimatarse a los climas cálidos. La falta de aclimatación que, en estos momentos, era vista como imposibilidad de adaptación de algunos pueblos a los climas tropicales, inclinaba la balanza de estos médicos hacia el esclavo africano o asiático como la única fuerza de trabajo. La excesiva importancia que se le concedía al clima, así como a la influencia que ejercía sobre las poblaciones, llevaron a los científicos a defender, hasta, al menos, la década de 1870 , la necesidad de importar mano de obra no blanca para realizar las tareas agrícolas. A pesar de considerar al hombre blanco el más inteligente y conveniente, en principio, para Cuba, pensaban que el clima influía en él de forma negativa, provocándole ciertas modificaciones fisiológicas que modificaban su carácter y le hacían caer en la indolencia. Por otra parte, el trato que debía darse a éstos ocasionaba gastos muy superiores a los de un esclavo o semiesclavo. A la vista de estas consideraciones, el negro africano era el trabajador más adecuado para el clima y faenas agrícolas de Cuba, si bien, apuntaba Dupierris, se debía comenzar a desterrar determinados criterios acerca de los chinos, que habían demostrado ser una fuerza de trabajo alternativa a la africana, siendo los únicos que tal vez pudieran sustituir a los negros. En su

5 Dupierris, M.: Memoria sobre la topografía médica de la Habana y sus alrededores y sobre el estudio físico y natural de los colonos asiáticos, La Habana, 1857. 
Memoria Dupierris desvelaba su intención de demostrar la viabilidad de la importación de trabajadores asiáticos como sustitutos de la mano de obra esclava:

“....tiene como objeto contribuir al plan de conducta necesario que debe seguirse, para que la colonización asiática, que el país reconoce ya muy útil y a propósito para los trabajos que constituyen su riqueza, preste todos los servicios de su inteligencia, de su actividad y hasta de su ambición...”. ${ }^{6}$

Otros estudios interesantes llevados a cabo sobre la anatomía y patología de las "razas" fueron los de Henri Dumont y Antonio Reynés de Verdier. Las investigaciones de Dumont sobre las patologías de las "razas" condujeron al médico francés a defender la idea de que existía una especial para cada pueblo, debido a la predisposición que cada "raza" tenía a adquirir una u otra enfermedad, así como a mantener que cada pueblo tenía una determinada inmunidad. ${ }^{7}$ En sus estudios Dumont, que basó parte de sus investigaciones en el análisis antropométrico de los distintos pueblos llegados a Cuba y Puerto Rico, se mostró contrario al mestizaje, por considerar que el "cruzamiento" ocasionaba siempre degeneración, la cual aumentaba con el influjo negativo del clima. La presencia de blancos en las Antillas, y sobre todo el trabajo de éstos en el campo, era en estos momentos una idea desestimada y desaconsejada por Dumont:

“...y mientras más siente un extranjero el influjo de la nueva zona climática que le domina, más pierde su riqueza orgánica de europeo, más se aproxima a las condiciones que favorecen o engendran la elefantiasis. Al lado del poder extenuante del clima se colocan las condiciones desfavorables del cruzamiento de las razas, de la mezcla de la sangre..., y el efecto negativo del cruzamiento, tan útil entre los individuos de la misma raza pura, va a parar en las Antillas a las monstruosidades hipertróficas...".

En contraposición a algunos de los criterios mantenidos por Dumont, en los trabajos de 1868 de Reynés, médico español y corresponsal en la Academia de La Habana, asistimos a la defensa de la diversidad de las

6 Ibidem, pág. 69

7 Dumont, Henri: Investigaciones generales sobre las enfermedades de la raza que no padecen de fiebre amarilla y estudio preliminar sobre la enfermedad de los ingenios de Azúcar, o Hinchazón de los negros y chinos, Cárdenas, 1865; "Antropología y Patología comparadas de los hombres de color africanos que viven en la Isla de Cuba”, Revista Bimestre de Cuba, ts. X y XI, La Habana, 1915-1916, págs. $161-420$ y $15-90$.

8 Dumont, Henri: Ensayo de una historia médico-quirúrjica de la Isla de Puerto Rico, La Habana, 1875. 
"razas", en cuya formación y transformaciones tenía una importancia decisiva el clima, la alimentación y las costumbres. La perpetuación de los nuevos caracteres adquiridos era posible a través de la herencia. Para Reynés el medio ambiente y el influjo que éste ejerciera sobre la población ocasionaba un "adelantamiento" de las poblaciones más atrasadas. ${ }^{9}$ Estudios como éste y los que a continuación expondremos ayudaron a sentar las bases que "legitimaron" y autorizaron desde un punto de vista científico la entrada de trabajadores blancos, muchos de ellos destinados a las labores del campo. La Sociedad Antropológica de la Isla de Cuba fue el otro gran escenario donde tuvieron lugar los debates de mayor interés y trascendencia para nuestro tema de estudio.

\section{La Sociedad Antropológica de la Isla de Cuba}

La fundación de esta Sociedad, el 26 de julio de 1877, supuso la institucionalización de la antropología y el desarrollo de diferentes investigaciones, las cuales tenían como eje central el estudio del hombre en todas sus dimensiones; su creación, además, supuso la apertura de un nuevo foro de debate de las teorías llegadas del viejo continente y Estados Unidos y de los trabajos de los científicos criollos. ${ }^{10}$ La Sociedad sirvió para canalizar y potenciar los trabajos e investigaciones que se venían realizando en Cuba sobre la conformación étnica del país, la diversidad de sus componentes étnicos, las modificaciones provocadas en determinadas poblaciones por la aclimatación, el surgimiento de nuevos grupos en función del cruzamiento, así como sus posibilidades de reproducción, la degeneración física de las "razas", la resistencia a las enfermedades y las patologías en función de su origen y adscripción étnica. Asimismo, se tendría en cuenta el estudio no

9 Reynés de Verdier, Antonio: "Algunas consideraciones generales sobre la raza negra. Su patología y terapéutica", Anales de la Real Academia de Ciencias Médicas, Físicas y Naturales de la Habana, t. 5, La Habana, 1868-1869, págs. 139-186.

10 Entre los estudios más relevantes sobre la Sociedad Antropológica de la Isla de Cuba y la ciencia antropológica caben destacar los realizados por Pruna, Pedro M. y García, Armando: Darwinismo y Sociedad en Cuba —Siglo XIX—, Madrid, 1989; García, A. y Pruna, A.: "El Transformismo en la Sociedad Antropológica de la Isla de Cuba”, Asclepio, vol. 39, núm. 1, Madrid, 1987, págs. 205-236; Rivero de la Calle, Manuel: Actas de la Sociedad Antropológica de la Isla de Cuba, La Habana,1966; García, Armando: Actas y resúmenes de Actas en la Sociedad Antropológica de la Isla de Cuba en publicaciones del siglo XIX, La Habana, 1988; Puig-Samper, Miguel Angel y Pelayo, Francisco: "Darwin en Cuba. El transformismo en la Revista de Cuba", Revista de Indias, núm. 186, Madrid, 1989, págs. 423-436. 
sólo físico de las "razas", sino también el moral, intelectual, psicológico y lingüístico, pero fundamentalmente centrado en las "razas" que poblaron y habitaban la isla. La gran diversidad de las cuestiones que se debatieron en la Sociedad hizo de ésta uno de los principales centros académicos del siglo XIX cubano en el que la antropología, la etnografía, la sociología, la arqueología, la etnología, la historia, la paleontología, etc. encontraron amplio desarrollo, y en el que participaron los más prestigiosos investigadores, algunos de los cuales eran a su vez miembros de la Real Academia de Ciencias Médicas, Físicas y Naturales de La Habana. ${ }^{11}$

En un principio la Sociedad Antropológica estuvo compuesta por una Junta directiva, integrada por Joaquín Jovellar, presidente; Juan Santos Fernández, vicepresidente; Gabriel Pichardo, tesorero vocal, y Luis H. Delmas, secretario general. El número limitado de socios, 24, fue aumentando considerablemente en los meses siguientes a la creación de la Sociedad, integrada por miembros de la elite intelectual, algunos de ellos directivos y seguidores del Partido Liberal Autonomista, como Luis Montané, Nicolás J. Gutiérrez, José Argumosa, Freire Andrade, Guillermo Willis, Ambrosio González del Valle, Antonio Govín y Torres, Julián Gassie, Enrique José Varona, José Antonio Cortina, José Rafael Montalvo, Antonio Mestre, Agustín W. Reyes, Arístides Mestre, Manuel Rafael de Castro, Vicente B. Valdés, José R. Torralbas, José Varela Zequeira, Juan Santos Fernández, Antonio Zambrana, J. Luis Dueñas y Felipe Poey, entre otros.

A mediados de julio de 1878 la Sociedad contaba con cinco Secciones: Gobierno, Antropología anatómica y prehistórica, Antropología fisiológica y patológica, Etnología y Ciencias auxiliares. Durante los primeros años, y hasta 1879, el órgano oficial de la Sociedad fue la Crónica Médico-Quirúrjica de La Habana; a pesar de que en 1879 comenzó a publicarse el Boletín de la Sociedad Antropológica de la Isla de Cuba, la Crónica Médico Quirúrjica continuó recogiendo en sus páginas los principales debates que tuvieron lugar en la Sociedad.

La introducción de nuevas "razas" y pueblos en Cuba provocó a lo largo del siglo XIX un rico discurso científico destinado, en un principio, a justificar la esclavitud y, posteriormente, a defender la inmigración y

11 Los discursos leídos en la sesión inaugural de la Sociedad Antropológica, el 7 de octubre de 1877, destacan la importancia de la antropología; véase: Poey, Felipe: "Discurso del Sr. Felipe Poey, Presidente de la Sociedad Antropológica de la Isla de Cuba", Boletín de la Sociedad Antropológica de la Isla de Cuba, t. 1, núm. 1, La Habana, 1879, págs. 8-9; Montané, Luis: "La antropología en Cuba. Consideraciones sobre el estado é importancia de la Antropología en la Isla de Cuba", Ibídem, págs. 10-16. 
colonización del país a base de familias blancas. ${ }^{12}$ Los argumentos contenidos en dicho discurso se superpusieron y, según las épocas, se moldearon de acuerdo a las necesidades de las elites económica y política. Las diferencias y barreras entre las poblaciones según su color y procedencia eran hechos constatables que podían analizarse a la luz de los conocimientos médicos, biológicos y antropológicos, y que determinaban, en última instancia, la posición que cada grupo debía ocupar en la escala social; una clasificación que justificaba el orden establecido y las diferencias, a todos los niveles, entre los individuos. $\mathrm{Y}$ es precisamente en la defensa del orden social y económico en la que la utilización de la ciencia adquirió un mayor significado, acomodándose a las circunstancias y cambios de las estructuras económico-sociales ocurridos en Cuba en el último cuarto del siglo XIX. De esta manera, de probarse que la aclimatación y adaptación a los trópicos no era monopolio de la "raza negra", y que ésta no tenía inmunidad a determinadas enfermedades, podría desarrollarse con éxito la incorporación del hombre blanco a los trabajos agrícolas de las Antillas, como lo manifestó José Argumosa en la sesión de la Sociedad del 2 de marzo de $1879 . .^{13}$

A partir de la década del 70, ya en plena descomposición del sistema esclavista, otros factores, que podían analizarse desde un punto de vista científico, comenzaron a tener importancia en el desarrollo de Cuba. Así, se iniciaron estudios sobre la diferente fecundidad entre las mujeres de color y las blancas, a fin de conocer si era o no real la necesidad de importar mano de obra; de demostrarse que las mujeres de color se reproducían más que las blancas, no sería necesario traer nuevos inmigrantes.

Por otra parte, la creencia generalizada de la existencia de una estrecha interrelación entre la "raza" y las enfermedades por la que se atribuía a individuos negros inmunidad frente a determinadas enfermedades, como la fiebre amarilla o el escorbuto, o bien su propensión a otros males, como la llamada "enfermedad del sueño", enfermedad endémica en algunas regiones africanas y que se pensaba que era una cualidad innata de la "raza" negra, así como la creencia de una aclimatación innata dada por las semejanzas de climas, fueron argumentos utilizados para justificar la existencia

12 García González, Armando y Naranjo Orovio, Consuelo: "Antropología, racismo e inmigración en la Sociedad Económica de Amigos del País de la Habana”, Asclepio, Monográfico dedicado a Ciencia y Sociedad en Cuba, vol. XLIII, fasc. 2, Madrid, 1991, págs. 139-164.

13 Acta de la sesión pública ordinaria del 2 de marzo de 1879, Rivero de la Calle: Actas de la Sociedad Antropológica ....., págs. 85-88. 
de la esclavitud. Las teorías sobre las que sustentaron dichos presupuestos fueron evolucionando en la medida que variaban las condiciones económicas y sociales. ${ }^{14}$ A ello contribuyeron los estudios que comenzaron a realizarse sobre los índices de natalidad y mortalidad de la población blanca y de color, que demostraron no sólo la falsedad de la teoría que otorgaba una mayor resistencia a determinadas enfermedades a las poblaciones según su procedencia y etnia, sino que probaron la capacidad de la población blanca para aclimatarse en las Antillas.

En esta misma línea se presentaron diferentes estudios a través de los que se trataba de demostrar los perjuicios e inconvenientes para el desarrollo y futuro de Cuba que causaba la continua introducción de trabajadores no blancos, y los beneficios que conllevaría la entrada de pobladores blancos. El trabajo de José Varela Zequeira titulado Estudios Estadísticos. Desnivel de los sexos en la Isla de Cuba, en 1882, relacionaba la desigualdad entre los sexos con la introducción de esclavos africanos y asiáticos, y expresaba la necesidad de auspiciar la colonización de la isla mediante familias blancas, que además de contribuir a equilibrar la balanza entre hombres y mujeres, con lo que sería posible un incremento de la población, ayudaría a mantener la "moralidad" en el país. ${ }^{15}$

En un gran número de debates se pusieron de manifiesto las concepciones que los científicos cubanos tenían en torno a la "raza", sinónimo de cultura, civilización y progreso. Para muchos de estos intelectuales la llamada "raza negra", los pertenecientes al tronco etíope, habían demostrado su incapacidad para generar civilización; una incapacidad que era inherente a ellos e independiente del medio (Montané, "La raza negra"). El afán por establecer categorías y jerarquías en la escala evolutiva y por probar científicamente que unos pueblos eran inferiores a otros provocó que dentro de la antropología se desarrollase con gran fuerza la antropometría.

14 La relación de las enfermedades con los temperamentos era un asunto que estaba presente desde la medicina hipocrática, y muchos médicos del XIX sí la aceptaron. Se creyó, por ejemplo, que el temperamento linfático predominaba en los negros y eso les predisponía a sufrir enfermedades como laringitis, tumores, elefantiásicos, trastornos oculares y parasitismo, trastornos que como vemos dependían de condiciones higiénicas en las que vivían. Véase los criterios del médico español Reynés de Verdier: "Algunas consideraciones...", págs. 139-148, 180-186; Chateausalins, Honorato Bernard de: El vademecum de los hacendados cubanos o guía practica para curar la mayor parte de las enfermedades, New York, 1831; Dupierris: Memorias sobre la topografía médica de la Habana y sus alrededores.....

15 "Acta de la sesión pública ordinaria del 6 de febrero de 1882", Actas de la Sociedad Antropológica..., pág. 125; Valdés Regués, Pedro: "Estudio Estadístico en que se compara la natalidad de la raza blanca, de la raza negra en los Estados Unidos de América", presentado en la Sesión del 21 de mayo de 1887, Ibídem, págs. 187-190. 
El inicio de un gran número de estudios de distintas poblaciones, a partir de los cuales fueron elaboradas tablas y medidas, motivó que los factores anatómicos y fisiológicos, como la estructura del cerebro, el peso y volumen del encéfalo, la capacidad craneana, la forma del cráneo (dolicocefalia, braquicefalia y mesocefalia) y el ángulo de Camper fueran elementos decisivos a la hora de establecer el grado de inteligencia de los pueblos. ${ }^{16}$

Otro de los grandes temas debatidos en la Sociedad Antropológica de la Isla de Cuba y en la Academia de Ciencias Médicas, Físicas y Naturales de La Habana fue el de la inteligencia de los pueblos, y en el que se hacía depender el grado de inteligencia y la capacidad intelectual de éstos con la "raza" a la que pertenecieran, sobre todo a partir de los estudios craneológicos de Morton. En Cuba fue un antropólogo y arqueólogo sevillano, Miguel Rodríguez Ferrer, quien siguió la clasificación de Morton para establecer categorías entre las distintas poblaciones que sucesivamente habían habitado la isla. ${ }^{17}$

La inferioridad de unas "razas" era un hecho que podía ser demostrado a partir de los estudios sobre el menor grado de inteligencia de los pueblos, encontrándose en los que ocupaban un lugar secundario en la escala evolutiva un cerebro de menor peso, volumen, y con una cantidad menor de sustancia gris y circunvoluciones. Seguidor de la antropología francesa, Enrique José Varona intentó demostrar que la masa encefálica de algunos europeos, alemanes o franceses, era superior a la de un hotentote en un treinta por ciento, a la vez que apuntaba otras diferencias en los hemisferios cerebrales de los primeros, con un número mayor de circunvoluciones y mayor sustancia gris. ${ }^{18}$

La antropometría y otros métodos fueron utilizados en los estudios que en estos momentos la antropología tenía como prioridad y entre los que

16 A partir de los criterios de Louis Pierre Gratiolet, Vacher de Lapouge o Clavel se establecieron diferencias entre las "razas" frontales superiores y las occipitales inferiores. Un trabajo que ilustra la aceptación de algunos de los conceptos discriminatorios de estos autores es la memoria de Mestre, Arístides: "La política moderna y la ciencia antropológica", Revista de Cuba, t. VI, La Habana, 1887, págs. 289-309. Para el caso de la utilización de los métodos antropométricos en la antropología norteamericana véanse los libros de Gould, Stephen: La falsa medida del hombre, Barcelona, 1984 y de Peset Reig, Ciencia y marginación.......

17 Rodríguez Ferrer, Miguel: Naturaleza y civilización de la grandiosa Isla de Cuba, Madrid, 1876-1887, pág. 132; Rivero de la Calle, Manuel y Puig-Samper Mulero, Miguel Angel: "Aportes de Miguel Rodríguez Ferrer a la Antropología cubana", Revista de Indias, núm. 194, Madrid, 1992, págs. 195-203.

18 Varona, Enrique José: "La moral positivista y la moral evolucionista", Revista de la Facultad de Letras y Ciencias de la Universidad de la Habana, Vol. III, La Habana, 1903, págs. 337-346. 
caben destacar aquéllos relacionados con los posibles problemas que originaba la convivencia entre distintos pueblos. La composición multiétnica de Cuba provocó una interesante discusión sobre la posibilidad o no de reproducción de los mestizos, que dividió a los miembros de la Sociedad entre los partidarios de las teorías más radicales de Gobineau y aquéllos, como Varona, que pensaban que el cruzamiento era beneficioso para la "raza" inferior. Los primeros, entre los que se encontraban Valdés Ragués, Mestre y Montalvo, mantenían la tesis de la incapacidad del mestizo para evolucionar y reproducirse más allá de la tercera o cuarta generación debido a ser individuos degenerados, que la propia selección se encargaría de eliminar. ${ }^{19}$ Estos científicos aplicaban al hombre la teoría de la hibridación que Darwin elaboró para los animales, según la cual no era posible la reproducción entre éstos después de la segunda o tercera generación, ya que consideraban al hombre negro una especie distinta del blanco. Para Montalvo la mezcla de las "razas" sólo producía degeneración, para lo cual se apoyaba en Darwin y citaba las observaciones de Humboldt sobre las deformaciones de las "razas" que se cruzaban y la longevidad de las "razas puras". ${ }^{20}$ En su discurso en torno a la influencia del cruzamiento de las familias, alababa las aristocracias de varios países considerándolas como los elementos que habían permitido conservar puras las "razas", e incluso llegaba a decir que un noble era "un producto de la selección bajo el doble aspecto físico y psíquico" (1 de abril de 1883). En el caso de Cuba comentaba que, de continuar existiendo la mezcla de "razas", lo que él llamaba "nuestra raza" iría descendiendo hasta desaparecer.

A similares conclusiones llegaba Arístides Mestre, quien, apoyándose también parcialmente en las tesis de Darwin, Spencer y Lapouge, aseguraba que la selección natural y social provocaba la supervivencia de las "razas" más aptas:

"Los pueblos no son más que aglomeraciones de individuos de razas más o menos homogéneas y a veces muy heterogéneas, con grados diversos de evolución y cualidad o aptitudes muy diferentes; la brillantez de las naciones no descansa más que en los caracteres de las razas que las constituyen. Mr. Lapouge expone que en Francia los artistas y poetas salen del tipo mediterráneo, así como también piensa que la raza

19 Gobineau, Joseph Arthur: Essai sur l'inegalité des races humaines, 4 vols., Paris, 1853-1855.

20 “Acta de la sesión pública ordinaria de 4 de marzo de 1883", Actas de la Sociedad Antropológica...., págs. 146-149. Véase, Naranjo Orovio y García González: Inmigración y racismo en Cuba en el siglo XIX... 
aria produce individuos activos que juegan el principal papel a los ojos del extranjero y ante la historia, haciendo para aquel autor la función de moléculas nerviosas, verdaderos cerebros del organismo social...." ${ }^{21}$

Por otra parte, en sus tesis sobresalía cierto determinismo biológico, que otorgaba a la herencia un papel fundamental a la hora de establecer categorías entre los pueblos, a pesar de lo cual dejaba abierta una posibilidad de cambio y "mejora" mediante la educación y el progreso. Para los seguidores de esta teoría, la herencia era el vehículo de transmisión de cualidades morales e intelectuales y de sentimientos, lo cual les permitía establecer una correlación entre la inteligencia, el carácter y la personalidad de los individuos y pueblos con los caracteres de las "razas".

La degeneración como producto del cruzamiento fue defendida por la gran mayoría de los científicos del momento. Así lo expresaba de forma rotunda José I. Torralbas en sus discursos y en su obra Los grupos satos en las razas humanas, en la que insiste en la inferioridad de los mestizos o "productos intermedios o satos". Desde un punto de vista etnológico el cruzamiento era calificado por este autor como algo negativo para la humanidad, generador de híbridos que poseían "una fusión de caracteres que los hace valer menos que el más inferior de los dos elementos que los ha producido". ${ }^{22}$

A estas tesis se opusieron, en parte, Armas y Varona en distintas sesiones de la Sociedad. Armas manifestó su opinión contraria a las emitidas sobre los cruzamientos y defendió que la heterogeneidad era el factor que hacía que los pueblos evolucionasen y se transformasen en pueblos poderosos, como lo habían demostrado algunos casos y, en particular, los romanos y americanos: "pues el cruzamiento de una raza con otras ha ido perfeccionando las castas de la misma manera que cruzando las mejores razas de animales se obtienen mejores crías". La selección natural se encargaba, según Armas, de que las "razas" inferiores no se perpetuasen ya que, afirmaba, como "vemos los mulatos no procrean más allá de una o dos generaciones". 23

Como ya apuntamos, Enrique José Varona lideraba una tesis menos radical y determinista que los anteriores, al sustentar que el cruzamiento entre dos "razas" era un hecho beneficioso para la inferior, que tendía a

21 Mestre: "La política moderna y la ciencia antropológica"...., págs. 289-309.

22 Torralbas: José I.: Los grupos satos en las razas humanas, La Habana, 1893.

23 "Acta de la sesión pública ordinaria del 6 de mayo de 1883", Actas de la Sociedad..., págs.155-158. 
desaparecer. Para llevar a cabo sus investigaciones sobre el proceso de adaptación y evolución cultural de las poblaciones y a fin de medir científicamente los hechos, Varona elaboró un Cuestionario sobre los niños de color, sus antecedentes étnicos, los grados de inteligencia y las cualidades de carácter. Dicho Cuestionario estaba restringido a los maestros de los niños de color — negros y mestizos-, y se ceñía a las asignaturas impartidas en las escuelas. El Cuestionario sería una forma empírica de hacer estadística en aras de la ciencia, y estaba orientado fundamentalmente a la observación de la evolución psicológica de estos niños. ${ }^{24} \mathrm{~A}$ fin de estudiar la conveniencia de su aplicación y llevarlo a cabo, se nombró dentro de la Sociedad una Comisión en la cual de nuevo aparecieron diferentes posturas. A pesar de los planteamientos científicos que sustentaba el Cuestionario hubo quienes se opusieron a su aplicación, como Vicente B. Valdés, al considerarlo "inoportuno e indeseable para la higiene moral". Finalmente, contó con el respaldo de muchos científicos, como Gassie, Reyes y Argumosa, para quienes sería una forma de de observar la evolución psíquica de los niños de "raza inferior", tanto en lo que se refería a la inteligencia como en lo relacionado con el carácter; por su parte Argumosa destacaba la importancia que tenía el hecho de estudiar una "raza" cuyo destino final era la emancipación, y para la cual debería ser preparada, "destituida como está de toda instrucción". ${ }^{25}$

En este sentido hay que destacar las tesis de algunos científicos que trataban de demostrar la superioridad física e intelectual de los negros criollos frente a los africanos (Reyes y Montalvo), derivada de la influencia del medio y del contacto de las "razas" inferiores con las superiores. Para Reyes la evolución, la herencia y el clima jugaban un papel decisivo en este proceso. Entre las causas que motivaban tales diferencias Reyes señalaba que el contacto con los blancos generaba en las "razas inferiores" ideas nuevas y "civilizadas", de las que hasta ese momento carecían, lo cual provocaba una modificación en sus cerebros y el desarrollo de facultades, todo lo cual podía ser transmitido por herencia a sus descendientes.

Por otra parte, el clima, considerado elemento capaz de modificar la "raza", contribuía a variar y "mejorar" los rasgos físicos, intelectuales y

24 “Acta de la sesión pública ordinaria del 7 de julio de 1878”, Actas de la Sociedad..., págs. 41-47. El Cuestionario elaborado por Varona era similar al "Cuestionario de Psicología Antropológica”, realizado por Letourneau en 1877 para la Sociedad Antropológica de Florencia.

25 "Acta de la sesión pública ordinaria del 4 de agosto de 1878", Actas de la Sociedad..., págs. 51-55. 
morales. Seguidor de la antropología francesa, cuyo dirigente más destacado pensaba que la capacidad craneana guardaba relación con el progreso intelectual de los pueblos, Reyes propuso llevar a cabo estudios comparativos entre adultos africanos y criollos, tales como medición y peso de cerebros, a fin de demostrar científicamente sus teorías. ${ }^{26}$

A una parte de estas afirmaciones y propuestas contestó Montalvo objetando la correlación entre el tamaño del cráneo y la inteligencia de los pueblos, ya que en muchos casos los cráneos mayores pertenecían a poblaciones menos evolucionadas, como era el caso de los negros africanos en Cuba. Para él, la capacidad intelectual dependía más de factores fisiológicos, como era el tamaño de los lóbulos cerebrales y el número de circunvoluciones, que del tamaño del cráneo. Asimismo, manifestaba que las diferencias entre los negros africanos y los negros criollos eran consecuencia del medio ambiente y de las relaciones que unos y otros tenían con otras poblaciones; en este sentido argumentaba que los negros africanos, residentes en el medio rural, eran inferiores debido a que no mantenían relación con otras poblaciones, mientras que los negros criollos, establecidos en las ciudades, presentaban un mayor grado de evolución debido a su contacto con la "raza" blanca. En cuanto al clima, Montalvo consideraba que éste no era un factor que influyera de forma tan determinante sobre las poblaciones como había manifestado Reyes.

Argumosa manifestó su conformidad con parte de las tesis expuestas por Montalvo, sobre todo en lo referente a la influencia que el medio ambiente y el contacto con "razas" superiores ejercía sobre las consideradas inferiores, fundamentalmente en el desarrollo de la inteligencia. ${ }^{27}$

La trascendencia que este tema tenía en Cuba mantuvo viva la polémica en la Sociedad Antropológica. La pregunta planteada por Antonio de León, en la sesión del 4 de febrero de 1883, sobre la influencia que ejercían las "razas" inferiores en los países habitados por la "raza caucásica" redirigía el debate hacia la conveniencia o no de continuar importando individuos no blancos en Cuba, o bien era necesario auspiciar la inmigración sólo de hombres blancos. En la discusión de nuevo cobró importancia el tema de la aclimatación, debido a la excesiva importancia que el orador concedía

26 Reyes, Agustín W.: "Estudio comparativo de los negros criollos y africanos", Boletín de la Sociedad Antropológica de la Isla de Cuba, t. 1, núm. 6, La Habana, 1879, págs. 130-135. Véase también "Acta de la sesión pública ordinaria del 12 de enero de 1879", Actas de la Sociedad Antropológica...., págs. 75-77.

27 Ibídem. 
al clima como factor determinante de la adaptación de las poblaciones, capaz de provocar cambios en el modo de ser físico u orgánico de éstas, pero no en cuanto a sus condiciones morales o intelectuales. Rechazaba el cruzamiento entre distintas "razas" ya que consideraba que, tan sólo, la presencia de "razas" inferiores en países habitados por otras superiores era perniciosa. ${ }^{28}$

La polémica estaba servida y la respuesta de Montalvo no se hizo esperar. En su argumentación éste explicó con algunos ejemplos históricos que el clima no era un factor que influyera en la alteración de los caracteres de los pueblos, ni que pudiera afirmarse que las "razas" se extinguieran. En su discurso recordó algunas de las teorías antropológicas de Quatrefages, que establecían subrazas dentro de los troncos raciales, que venían a demostrar la existencia de caracteres psíquicos y físicos distintos en cada subraza, y por lo cual no podían hacerse generalizaciones sobre el carácter feroz o el salvajismo de una "raza", como lo hiciera León.

\section{Aclimatación y población}

Desde la creación de la Junta de Población Blanca, en 1818, la posibilidad y capacidad de aclimatación del hombre blanco a Cuba fue uno de los temas que más preocupó a los promotores de la inmigración y del "blanqueamiento" de Cuba. Algunas enfermedades consideradas endémicas de la isla, como la fiebre amarilla o el vómito negro, limitaban la llegada de colonos y favorecían la importación de esclavos africanos, a los cuales, como hemos indicado, se creía inmunes a determinadas enfermedades. Pero no fue hasta mediados del siglo XIX cuando se inició la publicación de estudios que trataban de demostrar la posibilidad de adaptación y aclimatación del hombre blanco a los trópicos. Parte de estos trabajos aparecieron en las Memorias de la Sociedad Económica de Amigos del País de La Habana; en algunos de éstos se daba la vuelta a los argumentos hasta el momento esgrimidos e inclinaban la balanza hacia las posibilidades de la llamada "raza trigueña", cuyas cualidades generaban una mejor adaptación. En las comparaciones entre los pueblos y su capacidad de aclimatación se llegó a decir que la "raza trigueña" era la más apta para traer a

28 "Acta de la sesión pública ordinaria de 4 de febrero de 1883", Actas de la Sociedad Antropológica...., págs. 145-146. 
Cuba, cuyas condiciones la aventajaba a la "raza aria", incapaz de trabajar en los campos cubanos y tropicales. ${ }^{29}$

La importancia que la higiene fue adquiriendo como medio de controlar la propagación y contagio de enfermedades y epidemias, fundamentalmente a partir de la década del 30 del siglo XIX, convivió con el excesivo papel que se le siguió otorgando a la influencia del ambiente en la producción de enfermedades. Los partidarios de la aclimatación del europeo al clima tropical apoyaban sus tesis en estudios realizados en los que se tenía en cuenta la adopción de determinadas medidas de carácter higiénico o estratégico, como la elección de ciertas regiones del país y épocas del año para llevar a cabo los asentamientos de colonos, con las cuales podían mitigarse, e incluso evitarse, el contagio de enfermedades. Con estos estudios los defensores de la colonización y del aumento de población blanca contrarrestaron las tesis sobre el fatalismo del clima, insano para los inmigrantes procedentes de latitudes frías, $y$, sobre todo, la creencia generalizada de que las poblaciones negras eran las más capacitadas fisiológicamente para trabajar y vivir en los climas tropicales. Asimismo, se pensaba que el clima influía de forma negativa en las poblaciones y que, por ello, en las zonas tropicales los habitantes adolecían de cierta apatía, provocada por el clima. ${ }^{30}$

La importancia que se le concedía al medio ambiente y, en especial, al clima llegaba a tal extremo que se pensaba que éste imprimía al hombre caracteres especiales, que estaban en consonancia con las condiciones climatológicas del país que habitaban. Estas teorías y, en concreto, la que mantenía que el organismo del individuo sufría modificaciones en relación con el medio en que vivía fue mantenida por algunos científicos españoles, como fue el caso de Ramón Hernández Poggio. Desde un punto de vista lamarckista Hernández Poggio planteaba en su obra Aclimatación e higiene de los países europeos en Cuba, de 1874, que la aclimatación era un pro-

29 Véase el artículo traducido por Miguel Pons Guimerá, del médico francés Bordichon: "Inmigración de los países cálidos", Memorias de la Sociedad de Amigos del País, t. 44, La Habana, 1853-1854, págs. 271-285.

30 Para Pedro Armando Dufau, autor del libro De la abolición de la esclavitud colonial, el clima no era el factor responsable de la ocupación de determinados trabajos sólo por población de color. Entre las causas que motivaban este hecho Dufau señalaba la creencia generalizada de que determinados trabajos, relacionados con las labores del campo, eran degradantes y envilecidos y tenían que ser realizados por esclavos. La obra de Dufau está reproducida por Arango; véase Arango y Parreño, Francisco: Obras del Excmo. Señor D. Francisco de Arango y Parreño, t. 1, La Habana, 1888, págs. 659-750. 
ceso individual, que se producía de forma gradual. ${ }^{31}$ Según él, la aclimatación de los europeos al trópico era posible si se hacía gradualmente, de tal manera que el individuo hiciera escalas en zonas con temperaturas similares a la del país al que se dirigía. Estas escalas permitirían que el organismo se fuera lentamente adaptando al nuevo medio y se produjera la transformación:

“...la aptitud para la aclimatación depende del temperamento del emigrante; por lo tanto, el conocimiento de aquél y del punto que van a habitar, deben constituir la cuestión preliminar al tratarse del cambio de clima...

La disposición orgánica del emigrante influye mucho en los efectos sensibles que debe experimentar su constitución y el tiempo que durará dicho cambio..”. ${ }^{32}$

Como otros autores de la época, Hernández Poggio conocía la importancia de sus estudios y la aplicación de sus teorías para el desarrollo de los países, por lo cual dedicó gran parte de su investigación a probar la posibilidad real de la aclimatación de los europeos a las Antillas, y, fundamentalmente, las medidas higiénicas que habrían de seguirse. En su trabajo, tras un estudio minucioso de la geografía y clima de Cuba, comentaba algunas de las normas higiénicas y de alimentación que debían seguirse para lograr la aclimatación del europeo a la isla, así mismo indicaba que era necesario que el emigrante hiciera escala y permaneciera algunos días en lugares con climas similares, como Málaga o Tenerife:

“... todo emigrante debe graduar la transición de un clima a otro por una permanencia prolongada en climas parecidos al que va a habitar.

Siguiendo este importante precepto, la organización humana se adapta gradualmente a las modificaciones climatológicas diferentes a las que estaba habituado.

... Los climas mejores que conozco por sus cualidades y que más se aproximan a los tropicales, son: Santa Cruz de Tenerife, en las Islas Canarias, y Málaga”. ${ }^{33}$

Para demostrar la veracidad de su tesis Ramón Hernández Poggio ponía el ejemplo de la adaptación de los canarios a Cuba y Puerto Rico, cuyas costumbres, clima y vestimenta habían facilitado el cambio orgánico, que en cierta manera les hacía inmunes a determinadas enfermedades sufridas por los blancos cuando llegaban a estos países. La epidemia de fie-

31 Hernández Poggio, Ramón: Aclimatación e higiene de los países europeos en Cuba, Cádiz, 1874.

32 Ibídem, págs. 52-53.

33 Ibídem, págs. 62-63. 
bre amarilla que asoló Cuba en 1834, que causó el fallecimiento de sólo un $6 \%$ de los canarios que la habían contraído, evidenciaba la inmunidad de éstos a ciertas enfermedades.

En esta interesante polémica hay que destacar la contribución hecha por el médico naval español, Ángel Fernández-Caro y Nouvillas, quien dedicó gran parte de sus estudios a explicar cómo se llevaba a cabo la aclimatación del individuo y cómo, en definitiva, la adaptación del hombre blanco a climas tropicales era posible. ${ }^{34}$ En sus trabajos, Fernández-Caro y Nouvillas indicaba la importancia de la aplicación de estas investigaciones a las necesidades políticas y económicas de España, resaltando en ellas la capacidad del pueblo español para establecer colonias en otros países y latitudes como lo estaban haciendo las grandes potencias europeas. Para este científico la aclimatación era una cualidad de la humanidad y de determinados pueblos, y no del individuo. Según su tesis, el hombre no nacía aclimatado a todos los países, por lo que el "cosmopolitismo" no era una cualidad inherente a él, pero sí lo era respecto a la humanidad. El "cosmopolitismo" y la aclimatación se lograban tras años y siglos de continuo cruzamiento entre distintos pueblos, lo cual generaba la aparición de una "nueva raza", y en este caso superior:

"La influencia del medio empieza a ejercerse tan pronto como el individuo cambia las condiciones que lo rodean. Razas nuevas se forman sin más modificaciones que las del medio geográfico... Véase el criollo de las Antillas, producto inmediato de individuos de una misma raza, pero extraños al suelo tropical y obsérvese su color, la proporción de sus extremidades, la conformación de los pies y de las manos, multitud de detalles, que sin apartarlo del tipo primitivo, hacen de él una sub-raza con caracteres propios y, lo que es más admirable, con condiciones fisiológicas determinadas". ${ }^{35}$

Partidario de la unidad de la especie humana, Fernández-Caro defendía la superioridad de unos pueblos frente a otros; una superioridad que, en su opinión, era consecuencia de los cruzamientos y de la influencia del medio: "el blanco tiene condiciones de inteligencia de que carece el negro, así como cada raza presenta ciertas actitudes de que están privadas otras". ${ }^{36}$

34 Sobre la aclimatación véase la obra de Fernández-Caro y Nouvillas, Angel: El hombre y el clima, Discurso de recepción en la Real Academia de Medicina, Madrid, 1887, págs. 31-32, y "Estudios antropológicos", Anales de la Academia de Ciencias Médicas, Físicas y Naturales de la Habana, La Habana, tomo XIX, 1882-1883.

35 Fernández-Caro y Nouvillas: El hombre y el clima...., págs. 16-17.

36 Fernández-Caro y Nouvillas: "Estudios antropológicos", pág. 380. 
Para él, el mestizaje no era sinónimo o causa de degeneración, sino de "mejoramiento". Partiendo de estos presupuestos, Fernández-Caro defendía que la aclimatación era una facultad que dependía de las "razas", y no del individuo. Esta interesante tesis desembocaba en la defensa del mestizaje como proceso beneficioso a los pueblos, que habían ido cambiando sus condiciones genéticas y fisiológicas, adaptándolas a las nuevas condiciones y medios, y que hacían de éstos unos pueblos "superiores" capaces de aclimatarse a otras latitudes. Estos cambios beneficiosos producidos por la adaptación al medio se transmitían de generación en generación por la herencia.

Para el médico español la "raza" era el resultado de una serie de generaciones, de adaptación al medio, de aclimatación y de cruzamientos. El proceso de mestizaje del pueblo español, llamado por él "raza española", conferían al mismo unas ventajas insuperables para la colonización de otros países y, en concreto, para su asentamiento y adaptación a las Antillas. En su libro El clima y el hombre, editado en Madrid en 1887, Fernández-Caro defendía la superioridad de los españoles en los climas tropicales:

“A qué es debida esta superioridad climatológica de los españoles en los climas tropicales? Aquí, como en todo cuanto a aclimatación se refiere, encontramos la razón de la raza. Aunque indo-europea por su origen, la sangre española ha sufrido diversas fusiones con la sangre africana. Siro-árabes fueron los fenicios y cartagineses, primeros colonizadores de las costas meridionales de España, Portugal y Sicilia; moros procedentes de África dominaron durante ocho siglos toda la península ibérica, y hasta los lusitanos y cántabros (raza primitiva y autóctona en el suelo de la península, proceden, según ha demostrado el sabio filólogo Eschhoff por las afinidades del lenguaje, de la antigua Caldea, es decir, del África)". ${ }^{37}$

La importancia del clima como factor decisivo en la adaptación o no a latitudes tropicales motivó que se tratara de forma reiterada en distintas sesiones de la Sociedad Antropológica, en cuyo debate participaron muchos médicos y antropólogos. No sólo se trataba de hallar las causas científicas que permitieran establecer si la aclimatación era un fenómeno individual o colectivo, o si dependía de la "raza" del individuo, sino que se intentaba justificar por qué era conveniente, e incluso necesaria, la importación de trabajadores blancos. A modo de ejemplo citamos otra de las sesiones, que tuvo lugar en 1887, en la que otro científico se manifestó a favor de la adaptación y aclimatación del hombre blanco en los climas tro-

37 Ibídem, pág. 32. 
picales y en concreto de los españoles, defendido por Carlos de La Torre. En contra de los planteamientos de Orgeas, quien negaba la posibilidad de que un pueblo se aclimatase, La Torre - seguidor de Darwin- mantenía que la aclimatación era un caso particular de adaptación, que se lograría tras algunas generaciones. En sus conferencia se refirió de forma especial al pueblo ibero y a la capacidad del mismo de adaptarse al clima tropical. ${ }^{38}$

Dentro de los estudios realizados en Cuba sobre el clima hay que mencionar los de Carlos J. Finlay, que contribuyeron a desterrar las ideas sobre la benignidad y/o permisividad de determinados climas y a asentar la teoría sobre la posibilidad real de aclimatación del hombre blanco a los climas cálidos ${ }^{39}$ Como ponente de la Comisión que juzgaba el trabajo de Ramón Hernández Poggio, aspirante a un premio en la Real Academia de Ciencias Médicas, Físicas y Naturales de La Habana en 1874, elaboró un informe en el que rebatía parte de los argumentos del aspirante sobre la insalubridad del clima cubano, cuyas características propiciaban la aparición de enfermedades y epidemias. Para ello, Finlay se apoyaba en las estadísticas de mortalidad de la "raza" blanca, entre 1870 y 1874, de la ciudad de La Habana, y las comparaba con las de otras ciudades europeas y americanas, en las cuales la proporción de muertes era superior a las ocurridas en la capital cubana. ${ }^{40}$

Tres años después, Finlay volvía a presentar otras investigaciones en la Academia que sustentaban la tesis mantenida sobre la posibilidad de adaptación del hombre blanco a las Antillas y los beneficios de la colonización blanca. Una vez más las estadísticas demostraban la escasa mortalidad de la población blanca en Cuba, a pesar de estar constituida en una sexta parte por individuos procedentes de climas templados o fríos; asimismo, las tasas de mortalidad de los blancos - 22 por 1.000- eran inferiores a las registradas en otras poblaciones, sobre todo entre los asiáticos - que alcanzaban un 67 por 1.000 - cuyas costumbres "inmorales"-señalaba Finlay - eran motivo de su elevada mortalidad. Finlay consideraba que sólo estos datos podían ser tomados como fundamentos científicos en los que basar los proyectos de colonización e inmigración blanca.

38 "Acta de la sesión pública ordinaria de 6 de noviembre de 1887", Actas de la Sociedad Antropológica...., págs. 191-194.

39 Finlay, Carlos J.: "Apología del clima de Cuba", Gaceta Médica de la Habana, año I, núm. 2, La Habana, 1878, págs. 1-3.

40 Finlay, Carlos J.: "Informe acerca de la memoria Aclimatación e higiene de los europeos a Cuba, presentada con opción a uno de los premios de la Real Academia”, Anales de la Real Academia de Ciencias Médicas, Físicas y Naturales de la Habana, t. 2, La Habana, 1875, págs. 66-84. 
Con respecto a la población negra, con una tasa de mortalidad muy superior a la registrada en la blanca, y que además presentaba índices más altos entre la población libre que entre la esclava, Finlay aseguraba que ésta no reunía las condiciones adecuadas para reproducirse en Cuba, rechazando de esta manera la idea sustentada por otros científicos por la que hacían depender la aclimatación del hombre blanco del cruzamiento con poblaciones de color. ${ }^{41}$

\section{Una sociedad homogénea y civilizada}

La pluralidad étnica de la isla pesó en las concepciones y proyectos de los intelectuales y políticos, para quienes la homogeneidad cultural y étnica era sinónimo de civilización. Dicha homogeneidad sólo podría alcanzarse mediante la consolidación de una única "raza", considerada, por otra parte, bastión imprescindible en la formación de un pueblo y una nación.

En estos momentos la "raza" no sólo era una categoría social, sino que fue instrumentalizada y convertida en uno de los principales elementos de configuración de la nacionalidad cubana en formación. Para estos intelectuales, algunos de ellos políticos, la cubanidad, como lo era para José Antonio Saco, estaba restringida a un grupo concreto, compuesto exclusivamente por blancos. Los límites de la nacionalidad cubana seguían estando marcados en función del color de la piel, que además era símbolo de cultura. Por tanto, los portadores de la cubanidad, como pilar de la nacionalidad, tenían que ser, ahora como entonces, blancos.

Por otra parte, la descomposición del sistema esclavista aceleró el proceso y la puesta en marcha de forma más activa de una política de inmigración y colonización blanca. Por vez primera, en dicha tarea colaboraron desde sus distintos campos intelectuales, hacendados y políticos. Se hacía hincapié en la conveniencia de que la inmigración fuera libre y espontánea, con escasa intervención del Estado. Pero la apertura de la inmigración en Cuba hacía albergar ciertos temores que resucitaban el fantasma de la "negritud". Si bien era necesaria y urgente la entrada de mano de obra barata y abundante, había que establecer los límites en la entrada y controlar la posible llegada de "indeseables", como asiáticos o inmigrantes de color

41 Finlay, Carlos J.: "Breves observaciones del Dr. Juan Espada", Gaceta Médica de la Habana, núm. 5, La Habana, 1879, págs. 68-72. 
procedentes de Jamaica, Haití y África. Con ello se inició un proceso en el que la política inmigratoria estuvo presidida por criterios de selección étnica que se mantuvieron en las tres primeras décadas, años centrales de inmigración en Cuba en el siglo XX. ${ }^{42}$

El miedo a la "africanización" de Cuba, presente a lo largo de todo el siglo XIX, continuó en las últimas décadas de siglo, siendo una amenaza no sólo para el bienestar de la elite, sino también para el orden político. La participación de esclavos y gentes de color en la primera guerra de independencia evidenció a la metrópoli la necesidad de reforzar con elementos humanos su presencia y poder. La creación de colonias agrícolas y de colonias militares, así como la entrada de inmigrantes españoles, sin duda contribuirían a cumplir parte de los objetivos que España se propuso tras el Zanjón. La presencia mayor de españoles ayudaría a que la llamada "raza latina" terminara por absorber a la "negra". Si para muchos intelectuales la población de color era un elemento que impedía el avance y el progreso y frenaba la formación de una sociedad civilizada, para otros era, además, un elemento perturbador del orden.

El fin de la esclavitud, el crecimiento de la industria azucarera, la expansión económica y la necesidad de mano de obra fueron otros elementos que hay que tener en cuenta en el análisis de la sociedad y corrientes de pensamiento de la época; una época en la que la formación de la nación cubana confluyó con el cambio de las estructuras sociales y económicas que alteraron el sistema de producción, modificaron sus medios y sentaron las bases de una nueva sociedad. En este nuevo contexto se formaron distintas sociedades con el fin de auspiciar y fomentar la entrada de colonos e inmigrantes, que actuaron como intermediarias entre los hacendados y los colonos, como la Sociedad de Colonización de 1872, la Comisión Central de Colonización, que desde 1878 tenía unas atribuciones parecidas a las de la Junta de Fomento, y el Círculo de Hacendados de la isla de Cuba, también de $1878 .{ }^{43}$

42 Pérez de la Riva, Juan: El barracón y otros ensayos, La Habana, 1975; "Los recursos humanos de Cuba al comenzar el siglo: inmigración, economía y nacionalidad (1899-1906)", La república neocolonial. Anuario de Estudios Cubanos, t. 1, La Habana, 1975, págs. 7-44; "Cuba y la migración antillana 1900-1931”, La república neocolonial. Anuario de Estudios Cubanos, t. 2, La Habana, 1979, págs. 1-48. Sobre el control que ejercieron algunos científicos, sobre todo los médicos, en la sociedad y en la política cubana, y en concreto en la legislación sobre inmigración en las primeras cuatro décadas del siglo XX en Cuba, puede consultarse el libro de Naranjo Orovio y García González, Medicina y racismo en Cuba....

43 Archivo Histórico Nacional, Madrid (AHN), Ultramar, Cuba, Fomento, Leg. 174, exp. 1, doc. 1. 
Este contexto, en el que se combinaron diferentes factores, desembocó en la puesta en marcha con gran fuerza de diferentes proyectos de inmigración y colonización. El "hecho demográfico" comenzó a tomar nuevas dimensiones a partir de este momento, en el que fue instrumentalizado por políticos e intelectuales como uno de los elementos fundamentales en la consecución de sus fines y en el logro de una sociedad moderna y civilizada; una sociedad sobre la que sentar las bases de un Estado nacional, en el que la población de color continuó siendo marginada. Esta política de colonización e inmigración, ahora auspiciada desde el Estado e instituciones privadas, algunas de las cuales fueron creadas para tal fin, si bien era heredera en parte de la mantenida a lo largo del siglo, cobró una mayor fuerza y contenido cultural, político y social que, en gran medida, determinó que la ruptura con España no significara el fin de la afluencia de españoles a Cuba. A partir de 1898 la corriente inmigratoria española en la isla no sólo permaneció, sino que se incrementó, alcanzando cotas hasta ese momento desconocidas. Las causas no fueron sólo económicas, sino también sociales y culturales.

La medicina, la antropología y la biología continuaron marcando las normas que dictaban qué poblaciones eran deseables y cuáles no; quiénes eran los aptos y quiénes debían ser excluidos. Estas ciencias continuaron dando el contenido al discurso racial que presidió la política inmigratoria del siglo XX. La diversidad étnica y cultural continuó siendo considerada un factor de desintegración y no de riqueza, el miedo al "otro" entrañó nuevas fórmulas bajo preceptos médicos y sanitarios que calificaron a las poblaciones no "deseables" como inmigraciones "antisanitarias", a las cuales también se les acusó de ser un elemento que favorecía la desintegración nacional. Apoyados en determinadas teorías pseudocientíficas y de acuerdo a la legislación, en aras de la unidad nacional, de la soberanía, de la integridad cultural y de la defensa de los trabajadores nacionales se excluyó a todo individuo que se apartara de la norma establecida durante tantos años. ${ }^{44}$

44 En otro trabajo hemos analizado el papel que los científicos, fundamentalmente los médicos, tuvieron en el diseño de la política inmigratoria cubana, así como la influencia de las teorías pseudocientíficas en su legislación. La "higiene racial" en Cuba cobró importancia a medida que avanzó el siglo XX y se adoptaron en la isla políticas emanadas de legislaciones extranjeras que, acordes con los sentimientos "nacionales" de la elite, limitaron la entrada de extranjeros "indeseables". En los controles sobre la entrada de extranjeros, ceñidos a las poblaciones no blancas, encontramos no sólo argumentos culturales, sino también biológicos, sobre todo genéticos y médicos, por los que se calificaba a dichas inmigraciones de antisanitarias. Véase, García, Armando y Naranjo, Consuelo, "Inmigraciones e 'higiene racial' en Cuba, 1900-1940”, Un Siglo de España: el Centenario 1898-1998, Ciudad Real (en prensa). 